\title{
Enhanced beam of protons in plasma gas for three systems (tokamak, Z-pinch and ICF)
}

\author{
Baida M.Ahmed, Khalid A.Ahmad and Riayhd K.Ahmad \\ Physics Department, College of Science, Al-Mstansiriyah University \\ Bagdad, Iraq \\ E-mail address: ahmed_naji_abd@yahoo.com
}

Keyword: plasma gas, energy loss, individual ions, correlated ions and interference.

\begin{abstract}
The interaction of fast beam of proton impinging on a plasma target is treated theoretically, since in general the number density of the beam ions $n_{b}$ is much smaller than the electron density $n_{e}$ of the plasma target. The interaction between proton clusters (collective and individual) with plasma gas is evaluated using the dielectric dispersion function Vlasove formalism both for single and correlated protons.

In present work interaction clusters for proton on three different systems (tokamak, Z-pinch and inertial confinement fusion (ICF)) were used at different thermal energy (1000, 20 and 300) (a.u) and densities of proton $\left(10^{13}, 10^{18}\right.$ and $\left.3 \times 10^{22}\right) \mathrm{cm}^{-3}$ at three velocities $(1,7.5$ and 35$)$ a.u. to study the effect of these parameters. Found that collective excitations give a small contribution to the energy loss of single ions, We obtain the best beams of the protons in the system (ICF) and at high rates $(0,0.2,0.4,0.6)$ increase with increasing density. This gives a good beam of plasma proton use in different applications such as metal alloying, surface treatment, implantation, surface analysis, sputtering, determination of geometrical structures of polyatomic ions in addition give information about a variety of atomic-collision phenomena.
\end{abstract}

\section{1- Introduction}

The understanding of energy loss is very important in many applications involving the investigation of materials with beams of energetic ions, such as radiology, plasma physics, and astrophysics, new nanotechnology, irradiation of quantum dots, proton beam writing (PBW) and welding of nanotubes by ion beams or proton irradiation of carbon nanotubes (CNT).

The energy loss of fast charged particles in plasma gas great interest because of its considerable importance for the study of the basic interactions of charged particles.

The problem of these interaction beams particles with plasma has been attracting more and more interest in the last years in connection inertial confinement fusion (ICF), Z-pinch and tokamak systems based on the use of heavy-ion beams [1].

Of the most important factors in the study interference effect on cluster energy loss, based on simple models, considered the case of high cluster velocities, where the main contribution to the interference effect is short-range excitation. Both theoretically and experimentally, that the energy loss travelled path length for a cluster of ions moving in a plasma important differences-usually called vicinage effects. The origin of this effect is the interference in the electronic excitations of the target due to the correlated motion of the penetrating ions [2].

The role of interactions proton in plasma included to describe the interference effect for low and high cluster velocities therefore using the dispersion function in term of dielectric function[3].

The present work consider the cluster stopping in a plasma due to interactions protons with electrons target, for a range of cluster velocities starting from the low to high velocity of the protons. The dielectric function model is used to account for the interference effects in both collective and individual electron excitations for three systems (tokamak, Z-pinch and ICF).

The main goal of the present work is to calculate energy loss, within a dispersion function of dielectric theory, the stopping power in high and low velocity and the interference effect in term collisionless plasma to individual and collective ions.[4] 


\section{2-Energy loss of correlated ions in plasma}

The Energy loss of ions penetrating dense plasmas is given by equations of stopping power $S \equiv-d E / d x$, [5] when a point particle move in medium as plasma with charge Ze and velocity $v$, described by a dielectric function in form wave vector $k$ and frequency $\omega,[6,7]$

$$
s \equiv z^{2} S_{\circ}=\frac{2(z e)^{2}}{\pi v^{2}} \int_{0}^{\infty}\left(\frac{d k}{k}\right) \int_{0}^{k v} \omega d \omega \operatorname{Im}\left(\frac{-1}{\varepsilon(k, \omega)}\right)
$$

Where $s$ o the dielectric function in medium of plasma define as,

$$
\varepsilon(k, \omega)=1+\left(\frac{k_{D}}{k}\right)^{2} W(\zeta)
$$

in classical dense plasma dielectric formalism describe the stopping power, and $W(\zeta)$ in dielectric function represented the plasma dispersion function, [8]

$$
\zeta=\frac{((\omega+i \gamma)) / \omega_{p}}{k / k_{D}}
$$

When the damping $(\gamma)$ take $\gamma \rightarrow 0$ for collisionless plasma.

$$
\zeta=\frac{\omega / \omega_{p}}{k / k_{D}}
$$

Here must use some useful quantities. Plasma frequency $\omega_{p}$, thermal velocity $v_{T}$, wave number $k_{D}$ and Debye length $\lambda_{D}$ give as,

$$
\omega_{p}^{2}=\frac{4 \pi n_{p} e^{2}}{m}, \quad v_{T}=\left(\frac{k_{B} T}{m}\right)^{1 / 2}, \quad \lambda_{D}=\left(\frac{v_{T}}{\omega_{p}}\right), \quad k_{D}=\lambda_{D}^{-1}
$$

$n_{p}$ is the density of plasma electron and $\mathrm{T}$ is the electron temperature, and atomic unit will be given in atomic units.

$w(z)=X(z)+i Y(z)$

Where

$$
\begin{aligned}
& X(z)=1-z \exp \left(\frac{-z^{2}}{2}\right) \int_{0}^{z} d x \exp \left(\frac{x^{2}}{2}\right) \\
& Y(z)=\left(\frac{\pi}{2}\right)^{1 / 2} z \exp \left(\frac{-z^{2}}{2}\right)
\end{aligned}
$$

Plasma dispersion function $w(z)$ start from derivation of the longitudinal electron capability for a nonrelativistic thermal electron gas. In this paper using this formula to study and to describe movement the individual and collective clusters ions in plasma.[9]

Therefore Eq.(2) becomes,

$$
\varepsilon(k, \omega)=1+\left(\frac{k_{D}}{k}\right)^{2}(X(z)+i Y(z))
$$

Solving eq.(8) numerically by writing a subroutine in Fortran-90 in present work.

Let $z=\frac{\left(\omega / \omega_{p}\right)}{\left(k / k_{D}\right)} \rightarrow \omega=\frac{k \cdot z}{k_{D} \cdot \omega_{p}}$

so,

$d z=\frac{d \omega}{\omega_{p}\left(\frac{k}{k_{D}}\right)}, \quad d \omega=\frac{k \cdot d z}{k_{D} \cdot \omega_{p}}$

Rewrite Eq.(1) by taking the imaginary part of dielectric function given in Eq.(8) as:

$$
\operatorname{Im}\left(\frac{-1}{\varepsilon(k, \omega)}\right)=\frac{\left(k / k_{D}\right)^{2} Y(z)}{\left[1+\left(k / k_{D}\right)^{2} X(z)\right]^{2}+\left[\left(k / k_{D}\right)^{2} Y(z)\right]^{2}}
$$

And stopping power given in Eq.(1) becomes,

$$
S_{\circ}=\frac{2 \omega_{p}^{2}}{\pi v^{2}} \int_{0}^{\infty} \frac{k^{3} d k}{k_{D}^{4}} \int_{0}^{v / v_{T}} z d z \times\left(\frac{Y(z)}{\left\{\left(\frac{k}{k_{D}}\right)^{2}+X(z)\right\}^{2}+[Y(z)]^{2}}\right)
$$


To evaluated the energy loss of single ion move in medium as plasma using the dielectric formula and integrate first on variable $\mathrm{k}$,

$S_{\circ}=$

$\frac{2}{\pi}\left(\frac{\omega_{p}}{v}\right)^{2} \int_{0}^{v / v_{T}} d z z Y(z)\left\{\ln \left(\frac{k_{\max }}{K_{D}}\right)+\frac{1}{4} \ln \left[\frac{\left(1+\frac{k_{D}^{2}}{k_{\max }^{2}} X(z)\right)^{2}+\left(\frac{k_{D}^{2}}{k_{\max }^{2}} Y(z)\right)^{2}}{\left[X(z)^{2}+Y(z)^{2}\right]}\right]-\right.$

$\left.\frac{X(z)}{2 Y(z)}\left[\arctan \left(\left(\frac{k_{\max }^{2} / k_{D}^{2}+X(z)}{Y(z)}\right)-\arctan \left(\frac{X(z)}{Y(z)}\right)\right)\right]\right\}$

According to Bohm and Pines theory the first part is the individual mode $\left(k_{c}<k<k_{\text {max }}\right)$ and the second part in the collective mode $\left(0<k<k_{c}\right)$. Where $k_{c}=k_{D}=1 / \lambda$, To avoid logarithmic divergences in this integral for large $\mathrm{k}$, a cut off $\left(k_{\max } \equiv 1 / b_{\min }\right)$ must be introduced. The origin of this cut off is due to the limit of applicability of the dielectric approach to treat short-range interactions.[10]

$$
S_{\circ}=\int_{0}^{k_{D}} f(k) d k+\int_{k_{D}}^{k_{\max }} f(k) d k=S_{\circ}^{c o l}+S_{\circ}^{\text {ind }}
$$

The stopping power has been solved numerically taking in the consideration an approximation for low and high velocity. A program Baida-for has been written in Fortran-90 for numerical solution, and a copy of program is a vailable in [to sumite on].

\section{Special cases}

\section{(a)At high velocity approximation $\left(v>v_{T h}\right)$}

Where $\mathrm{v}>>\mathrm{v}_{\mathrm{th}}$, where .vth is the thermal velocity[10 ]

$$
\operatorname{Im}(-1 / \varepsilon(k, \omega)) \cong \frac{\pi}{2} \omega_{p}\left[\delta\left(\omega-\omega_{p}\right)-\delta\left(\omega+\omega_{p}\right)\right]
$$

Then Eq.(13) become,

$S_{\circ}=S_{\circ}^{c o l}+S_{\circ}^{i n d} \approx\left(\frac{\omega_{p}}{v}\right)^{2}\left[\ln \left(\frac{v}{v_{T}}\right) \Theta\left(v-v_{T}\right)+\ln \left(\frac{k_{\max }}{k_{D}}\right) \Theta\left(k_{\max }-k_{D}\right) F_{1}\left(\frac{v}{v_{T}}\right)\right]$

Where $(\Theta)$ is Heaviside function [stegun, 1971]

$F_{1}(x)=\left(\frac{2}{\pi}\right)^{1 / 2} \int_{0}^{x} z^{2} \exp \left(\frac{-z^{2}}{2}\right) d z$,

Therefor,

$S \circ \cong\left(\frac{\omega_{p}}{v}\right)^{2} \ln \left(\frac{k_{\max }}{k_{\min }}\right)$

Where $k_{\min }=\omega_{p} / v=1 / b_{\max }$ represent the the dynamical parameter and determined by the dielectrical model. There are two expression for collisions, [10]

(i) Bethe expression represent the quantum mechanical value $\left(k_{\max }=2 \mathrm{mv} / \mathrm{h}\right)$ where the $\operatorname{logarithm}\left(\ln \left(2 m v^{2} / \omega_{p} \hbar\right)\right.$.

(ii) Bohr's expression represent the classical mechanical value $k_{\max }=m v^{2} / z e^{2}$ where the logarithm $\left(m v^{3} / z e^{2} \omega\right)$. 
The expression to transition between both cases:

$\ln \left[\frac{k_{\max }}{k_{\min }}\right] \cong \ln \left[\frac{\alpha v^{2}}{\left(1+\beta v^{2}\right)^{1 / 2}}\right]$

Where $\alpha=1.123 m v / z e^{2} \omega_{p}, \beta=\left(\hbar / \Gamma z e^{2}\right)^{2}, \Gamma=1.781$

(b) low velocity approximations $\left(v<v_{T}\right)$

In low velocity the electron-ion collisions controlling where the short-range excitations only take place, so use the approximation $z=\frac{\omega}{k v_{T}} \ll 1$ to the dielectric function [12].

$$
\operatorname{Im}\left(\frac{-1}{\varepsilon(k, \omega)}\right) \cong \sqrt{\frac{\pi}{2}}\left(\frac{k}{k_{D}}\right)^{2}\left\{\frac{z \exp \left(-z^{2} / 2\right)}{\left[1+\left(k / k_{D}\right)^{2}\right]^{2}}\right\}
$$

and if $k \ll k_{D}$ the approximation become:

$$
\operatorname{Im}\left(\frac{-1}{\varepsilon(k, \omega)}\right) \cong \sqrt{\frac{\pi}{2}} \frac{\left(\omega / \omega_{p}\right)}{\left(k / k_{D}\right)^{3}} \exp \left(\frac{-z^{2}}{2}\right)
$$

where the stopping power becomes,

$$
S \circ=\frac{1}{3}\left(\frac{2}{\pi}\right)^{1 / 2} \frac{1}{\lambda_{D}^{2}} L\left(\frac{k_{\max }}{k_{D}}\right)\left(\frac{v}{v_{T}}\right) F_{2}\left(\frac{v}{v_{T}}\right),
$$

where $\mathrm{L}(\mathrm{x})$ represents the low-velocity of collision logarithm

$$
L(x)=\frac{1}{2}\left[\ln \left(1+x^{2}\right)-\frac{x^{2}}{\left(1+x^{2}\right)}\right]
$$

and,

$$
F_{2}(x)=\frac{3}{x^{3}} \int_{0}^{x} z^{2} \exp \left(\frac{-z^{2}}{2}\right) d z
$$

Figure (1) shows the numerical variation of stopping number L and functions F1 and F2 with variable $\mathrm{x}$ in the program (Baida.for). As $x \rightarrow 0, F_{2}(x) \rightarrow 1$ and $x \rightarrow \infty, F_{2}(x) \rightarrow 0$, while $F_{1}(x) \rightarrow$ 0 as $x \rightarrow 0$ and $F_{1}(x) \rightarrow 1$ as $x \rightarrow \infty$ as shown in Fig.(1).

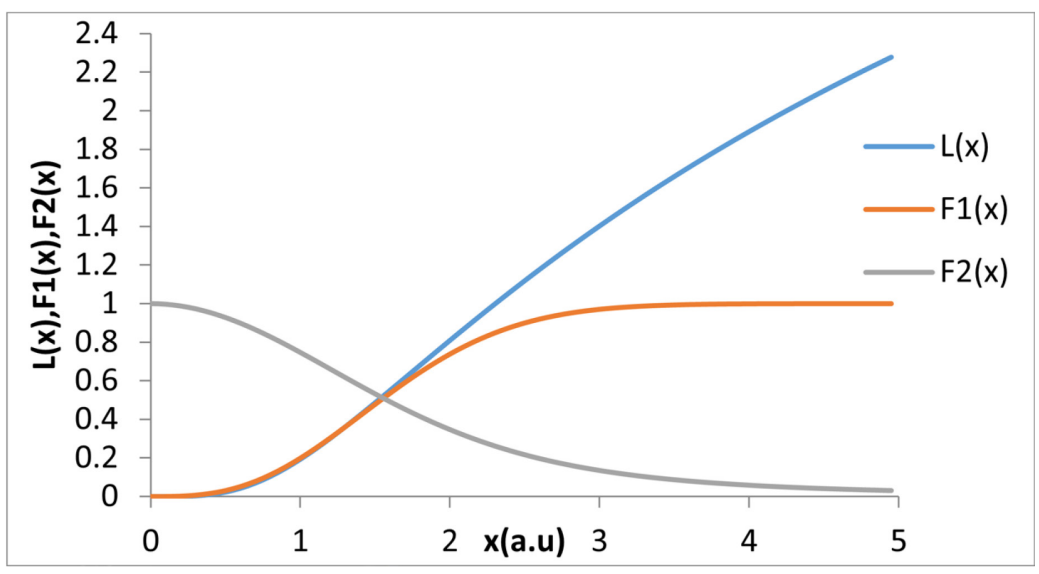

Fig.(1) The stopping power in high and low velocities for different equations (), that evaluated analytical approximation. 


\section{3-Interference effect on the energy loss}

The energy loss of a pair of charges in correlated motion (dicluster) shows some significant differences with the case of uncorrelated particles discussed before. These differences come from interference effects in the simultaneous interactions of both particles with the medium[13], and internuclear distances of several atomic units, the interferences are generally more important for the collective - interaction terms. Therefore, the role of collective terms in this case must be carefully reevaluated.

The stopping power of a dicluster can be expressed as follows:[14 ]

$$
S_{\text {dicluster }}=2 Z^{2}\left[S_{0}+I(r)\right]
$$

$I(r)$ is the interference function.

In terms of stopping power $S_{0}$, defined in eq. (1) and the interference function $I(r)$ given by [15],

$$
\begin{aligned}
& I(r)=\left(\frac{1}{2 \pi^{2} v}\right) \int d k\left(\frac{k \cdot v}{k^{2}}\right) \operatorname{Im}\left(\frac{-1}{\varepsilon(k, \omega)}\right) \cos (k \cdot r) \\
& \text { (i) } \quad r \rightarrow 0, \quad I(r) \rightarrow S_{0}, \quad \text { and } S_{c l} \rightarrow(2 Z)^{2} S_{0} \\
& \text { (ii) } \quad r \rightarrow \infty, I(r) \rightarrow 0, \quad \text { and } S_{c l} \rightarrow 2 Z^{2} S_{0}
\end{aligned}
$$

Using Eq.(11) for $\operatorname{Im}[-1 / \epsilon(k, \omega)]$ in Eq.(26), one can rewrite it as follows:

$$
I(r)=\frac{2 \omega_{p}^{2}}{\pi v^{2}} \int_{0}^{\infty} \frac{k^{3} d k}{k_{D}^{2}}\left(\frac{\sin (k r)}{k r}\right) \int_{0}^{v / v_{T}} z d z *\left(\frac{Y(z)}{\left\{\left(\frac{k}{k_{D}}\right)^{2}+X(z)\right\}^{2}+[Y(z)]^{2}}\right)
$$

The integration over the $k$ variable may be performed analytically. After some algebra, one can express the function $I(r)$ as a single integral.

$$
I(r)=\frac{2}{\pi}\left(\frac{\omega_{p}}{v}\right)^{2}\left(\frac{\lambda_{D}}{r}\right) \int_{0}^{v / v_{T}} z d z \operatorname{Im}\left[F\left(z, K_{\max }, r\right)\right]
$$

Where

$$
\begin{aligned}
& F\left(z, k_{\max }, r\right)=q\left\{\sin (q r)\left[\operatorname{Ci}\left(\left(k_{\max }-q\right) r\right)+\operatorname{Ci}\left(\left(k_{\max }-q\right) r\right)-\operatorname{Ci}(-q r)-\operatorname{Ci}(q r)\right]+\right. \\
& \left.\cos (q r) \times\left[\operatorname{si}\left(\left(k_{\max }-q\right) r\right)-\operatorname{si}\left(\left(k_{\max }+q\right) r\right)-2 \operatorname{Si}(-q r)\right]\right\}
\end{aligned}
$$

Then Eq.(28) becomes,[10 ]

$$
\begin{aligned}
& I(r)=\left(\frac{\omega_{p}^{2}}{v}\right)\left[I\left(\frac{\omega_{p} r}{v}, \frac{r}{\lambda_{D}}\right) \theta\left(v-v_{T}\right)+F_{1}\left(\frac{v}{v_{T}}\right) I_{1}\left(\frac{r}{\lambda_{D}}, k_{\max } r\right) \theta\left(k_{\max }-k_{D}\right)\right] \\
& =I_{(r)}^{\text {col. }}+I_{(s r)}^{\text {ind. }}
\end{aligned}
$$




\section{4- Systems}

\section{a. Tokamak system:}

A tokamak as shown in Fig.(2) is a device using a magnetic field to confine a plasma in the shape of a torus. Achieving a stable plasma equilibrium requires magnetic field lines that move around the torus in a helical shape. Such a helical field can be generated by adding a toroidal field and a poloidal field. In a tokamak, the toroidal field is produced by electromagnets that surround the torus, and the poloidal field is the result of a toroidal electric current that flows inside the plasma. This current is induced inside the plasma with a second set of electromagnets.[16]

The fusion reactions in the plasma spiraling around a tokamak reactor produce large amounts of high energy neutrons. These neutrons, being electrically neutral, are no longer held in the stream of plasma by the toroidal magnets and continue until stopped by the inside wall of the tokamak.[17]
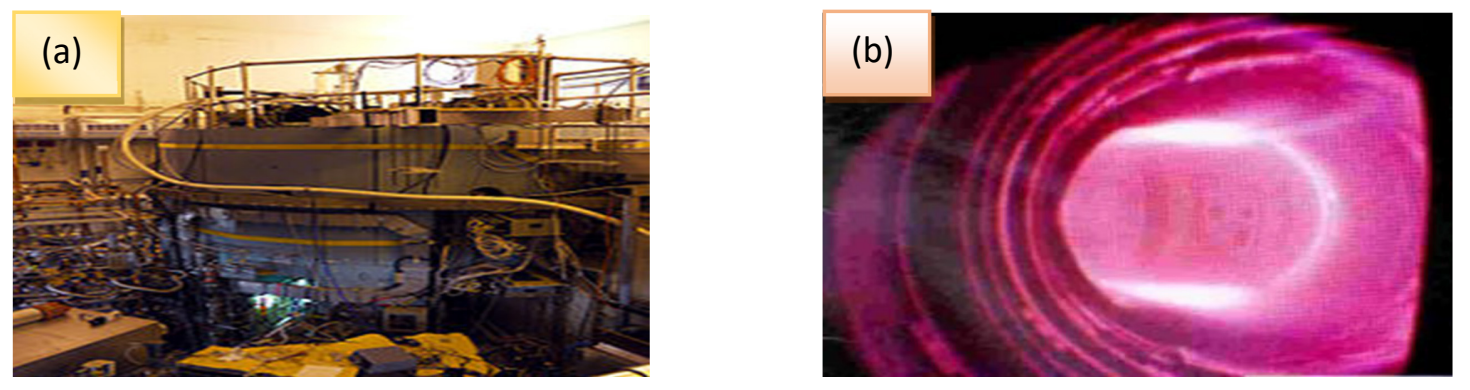

Fig.(2) (a) Show the tokamak system (b) the plasma formed within the system tokamak.[18]

\section{b. Z-pinch}

The Z-pinch is an application of the Lorentz force as shown in Fig(3), in which a current-carrying conductor in a magnetic field experiences a force. One example of the Lorentz force is that, if two parallel wires are carrying current in the same direction, Z-Pinch physics considered in the development of a simplified Z-Pinch fusion thermodynamic model to determine the quantity of plasma, plasma temperature, rate of expansion, and energy production to calculate parameters and characterize a propulsion system.[16]

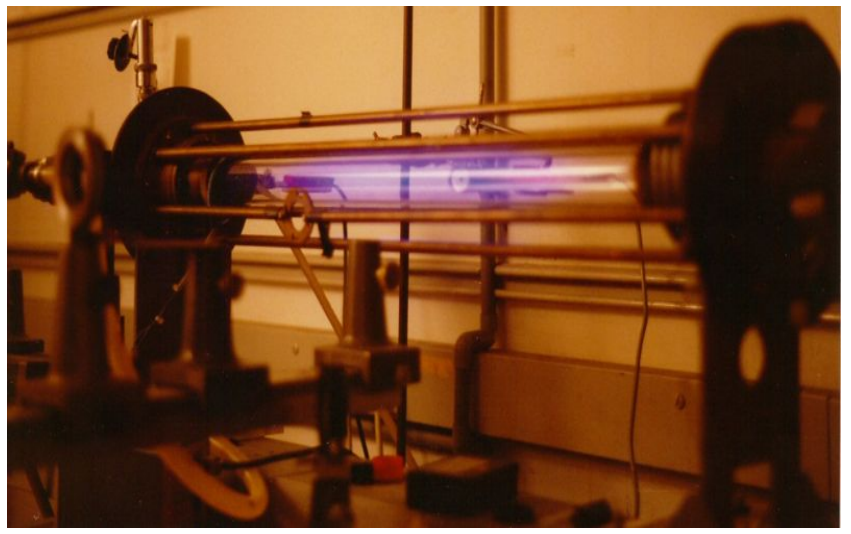

Fig.(3) Show the Z-pinch system[19] 


\section{ICF system}

In inertial confinement fusion (ICF) is shown in Fig.(4), a high density, low temperature plasma can be obtained during the compression phase, so minimizing the energy needed for compression. If the final temperature reached is low enough, the electrons of the plasma can be degenerate. In this case, bremsstrahlung emission is strongly suppressed and ignition temperature becomes lower than in classical plasmas.[7].

The obvious interest of using the heaviest ions in drivers for compressing ICF targets makes it desirable to also consider atomic clusters with arbitrarily large mass 1 . The production of clusters and their internal properties have been given a certain attention recently from the inertial thermonuclear fusion point [20]. The comparison between three systems is shown in table (1).

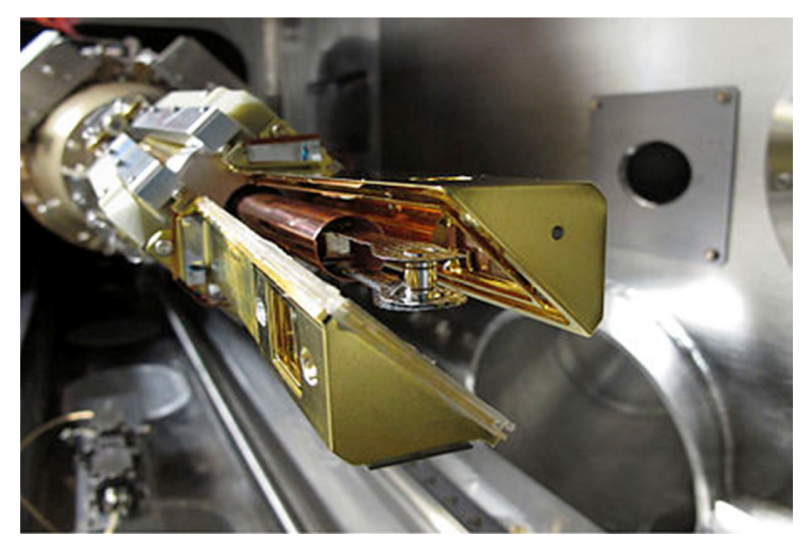

Fig.(4) Show the ICF system [20]

Table (1) Some important parameters using in this paper for Tokamak, Z-pinch and ICF evaluated by velocities (1, 7.5 and 3.5) a.u respectively.

\begin{tabular}{|l|c|c|c|}
\hline Parameters & Tokamak & Z-pinch & ICF \\
\hline $\mathrm{np}(1 / \mathrm{cm} 3)$ & $10^{13}$ & $10^{18}$ & $3 \times 10^{22}$ \\
\hline $\boldsymbol{\omega}_{\boldsymbol{p}}$ & $4.13 \times 10^{-6}$ & $1.36 \times 10^{-3}$ & 0.236 \\
\hline $\mathrm{T}(\mathrm{eV})$ & 1000 & 20 & 300 \\
\hline $\mathrm{v}_{\mathrm{T}}$ (a.u) & 6.06 & 0.857 & 3.32 \\
\hline$\lambda_{\boldsymbol{D}}$ (a.u) & $1.4 \times 10^{6}$ & 630 & 14.1 \\
\hline $\mathrm{b}_{\max }$ (a.u) & $2.32 \times 10^{5}$ & $5.5 \times 10^{3}$ & 148 \\
\hline $\mathrm{T} / \mathrm{T}_{\mathrm{f}}$ & $5.91 \times 10^{8}$ & $5.49 \times 10^{3}$ & 85.3 \\
\hline
\end{tabular}



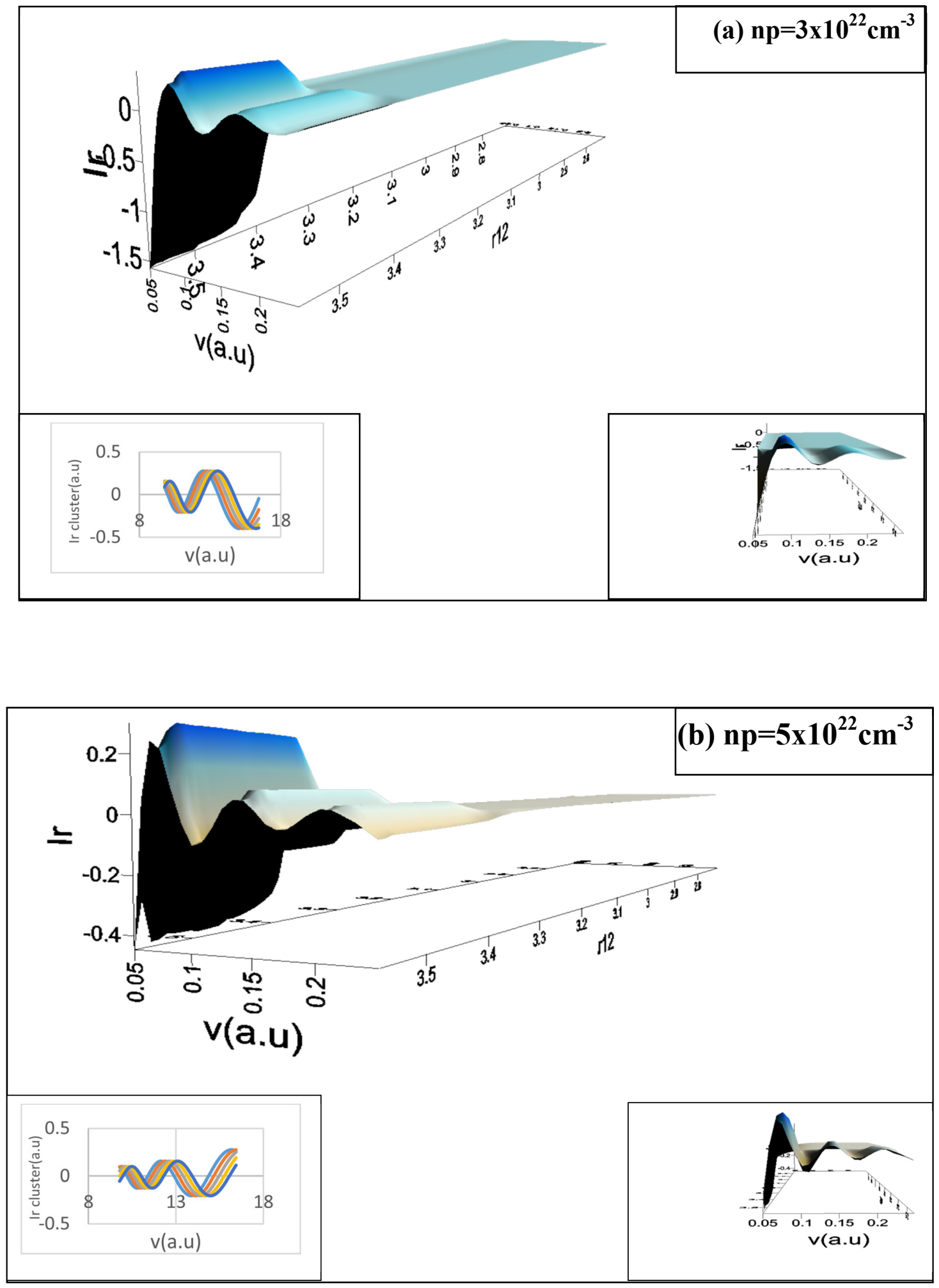

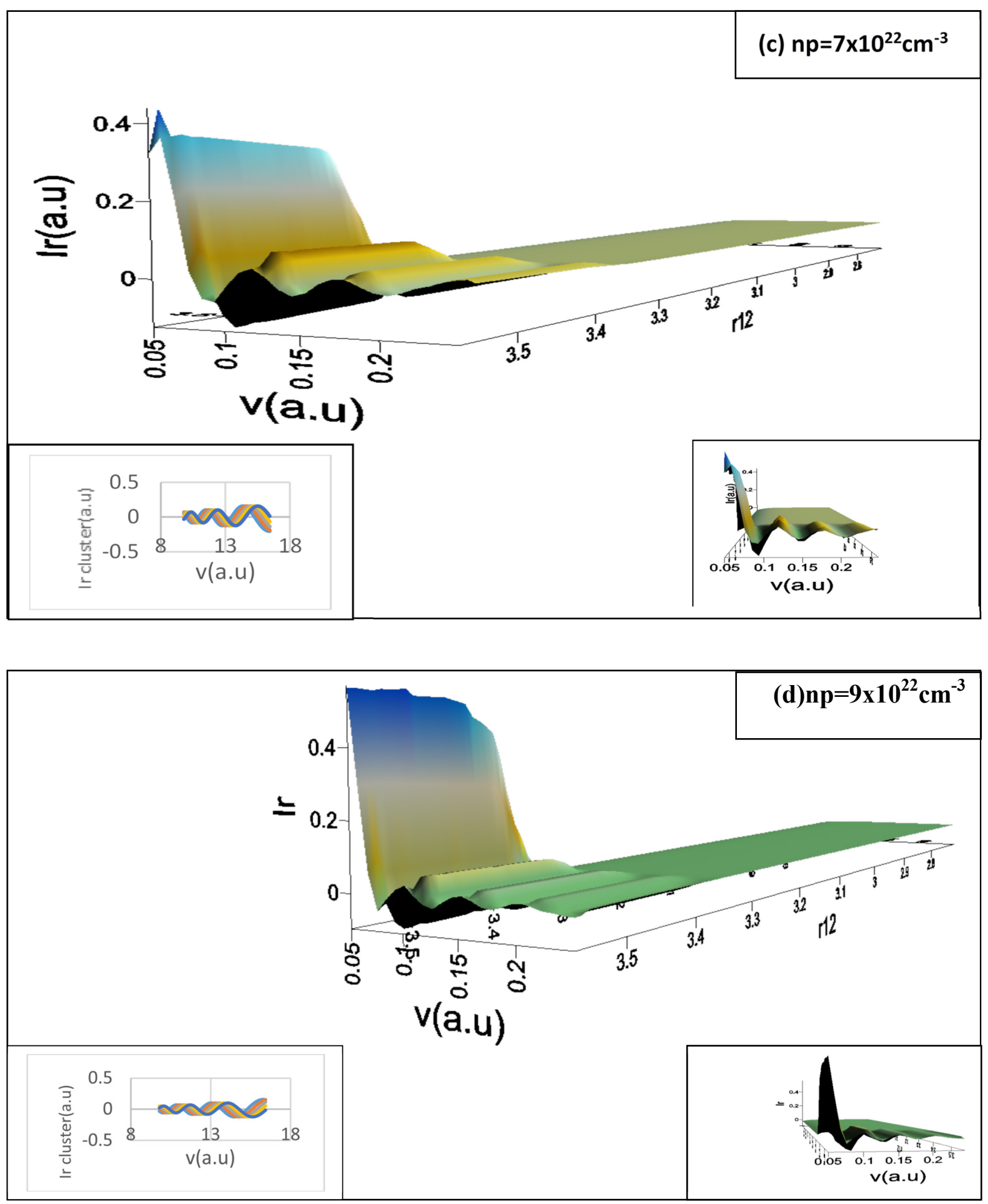

Fig.(5) The interference effect Ir with distance $r_{12}$ and projectile velocity $v$ in (a.u) on ICF system in different d ensities, (a) $n p=3 \times 10^{22} \mathrm{~cm}^{-3}$, (b) $n p=5 \times 10^{22} \mathrm{~cm}^{-3}$, (c) $n p=7 \times 10^{22} \mathrm{~cm}^{-3}$ and (d) $n p=9 \times 10^{22} \mathrm{~cm}^{-3}$ and the other parameters are in table (1). 

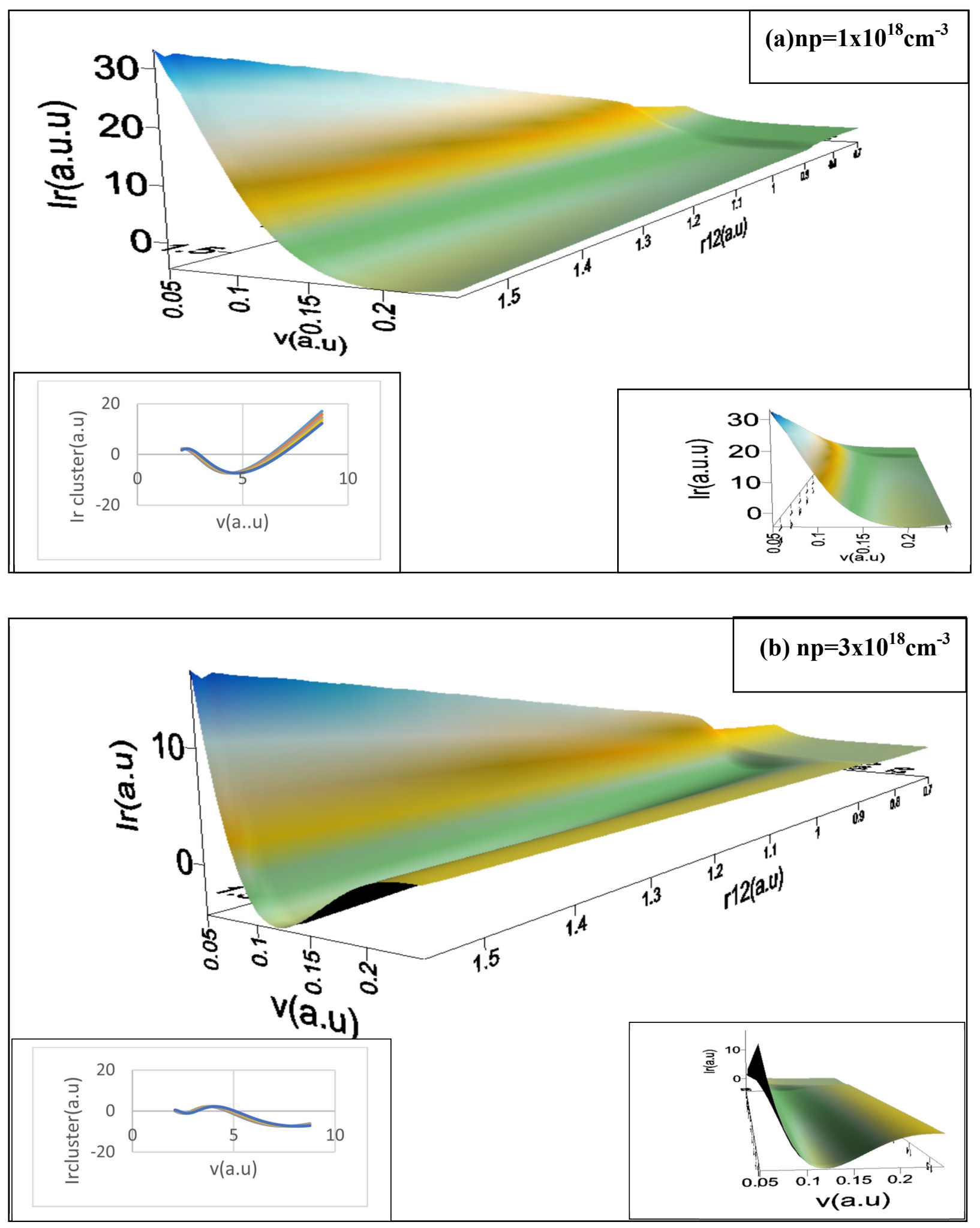

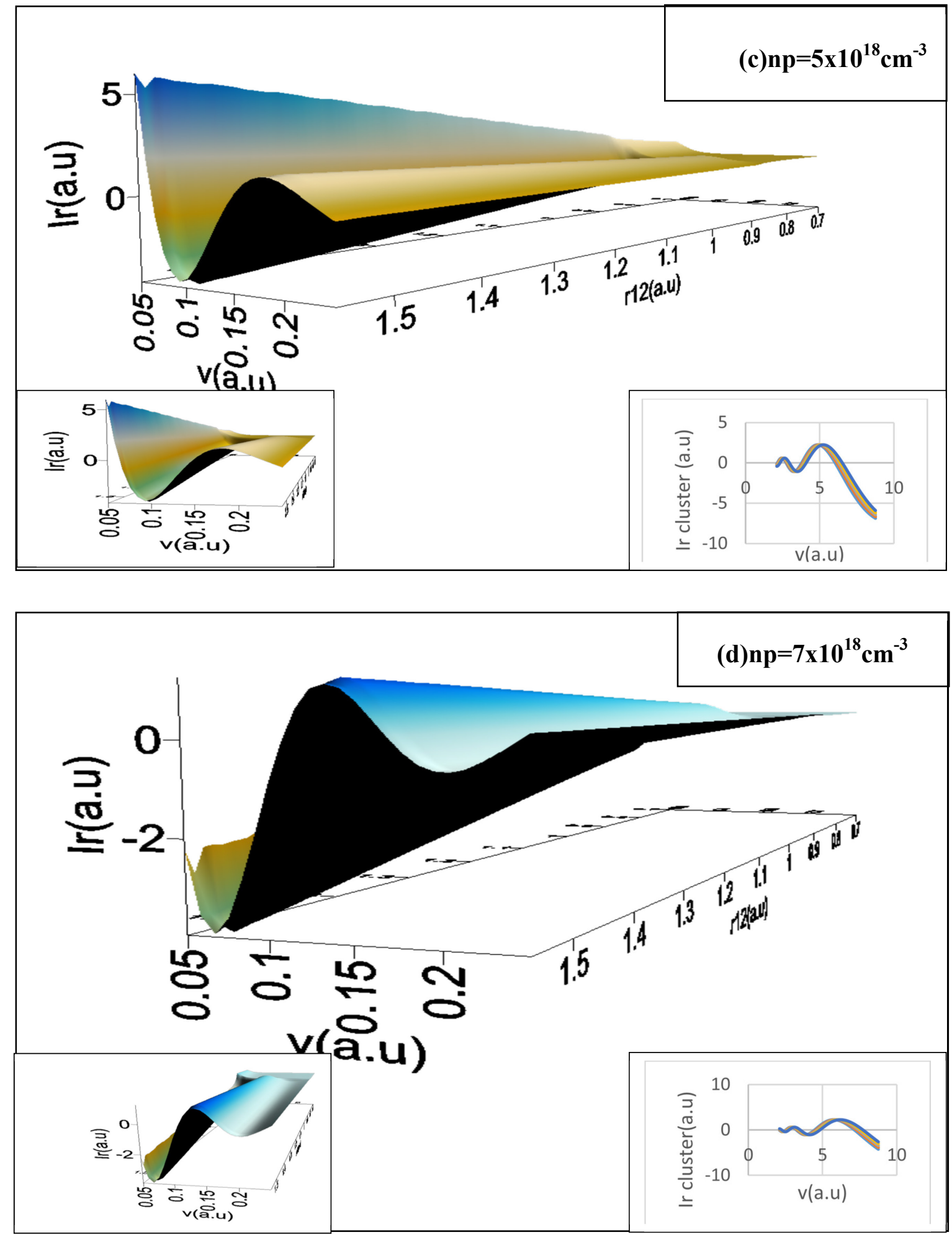

Fig.(6) The interference effect Ir with distance $r_{12}$ and projectile velocity $v$ in (a.u) on $Z$-pinch system in different densities, (a) $n p=1 \times 10^{18} \mathrm{~cm}^{-3}$, (b) $n p=3 \times 10^{18} \mathrm{~cm}^{-3}$, (c) $n p=5 \times 10^{18} \mathrm{~cm}^{-3}$, (d) $n p=7 \times 10^{18} \mathrm{~cm}^{-3}$. and other parameters in table (1). 

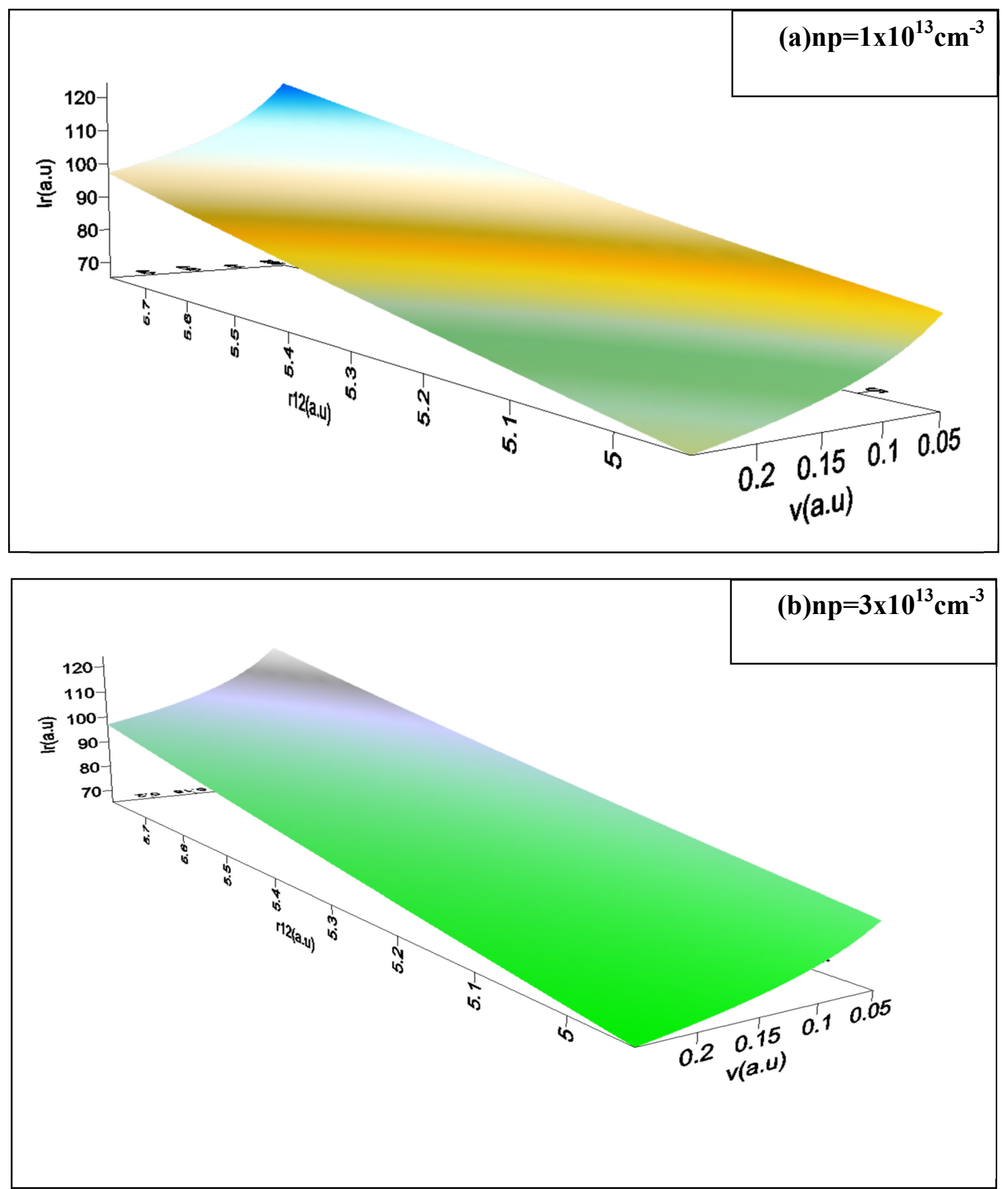

Fig. (7) The interference effect Ir with distance $r_{12}$ and projectile velocity $v$ in (a.u) on Tokmak system in different densities, (a) $n p=3 \times 10^{13} \mathrm{~cm}^{-3}$, (b) $n p=5 \times 10^{13} \mathrm{~cm}^{-3}$, and the other parameters are in table (1). 


\section{DISCUSSION}

The theory of heavy-ion stopping power is developed as a basis of the influence of the individual and collective clusters on the stopping in plasma, this stopping gives full concept on interactions the ions in plasma gas. We adopted use the dispersion function of dielectric formal in plasma and evaluated the equations numerically and analytical by using a software Compaq visual Fortran v6.6 for linking and executing programs. We study the ions stopping power for various classical and quantum plasma parameters, such as the number density, velocities, electron temperature, interference effect and effective range $\left(b_{\max }\right)$. And the basic factor between two cases (classical and quantum) the parameter $\mathrm{k}_{\max }$.

Figure (1) displays the results of analytical values for equations stopping that translator format forms. Where (F1) represent the individual excitations at high velocity in this case the Debye screening modified as the dynamical screening distance because the wake potential behind moving ion.

At low velocity $(\mathrm{L})$ represent the collective excitations in this case Debye screening represent by effective range $\left(b_{\max }\right)$, both cases the parameter $\mathrm{k}_{\max }$ and $\mathrm{k}_{\min }$ controls on stopping in plasma.

This study showed the individual excitations are best in the stopping from the collective excitations. Where the short-rang collisions control in high velocity that represents the quantum values. At low velocity close electron-ion collisions is the dominant and here shows the influence Coulomb potential.

Figures (5, 6 and 7) show the interference function at different velocities and internucler distance $r_{12}$, the difference between three systems (ICF, Z-pinch and tokamak) come from interference effect, this factor its more important in low velocity so the affective appear on collective interaction than individual. So the effect of density on the stopping where the number of folds increases with increase density because the relationship between the frequency and density in stopping equation (30). As well the contribution of collective modes increases with plasma density and decreases with temperature.

\section{CONCLUSIONS}

In presented work a stopping power of proton clusters penetrating plasma has been where studied numerically and analytical for group of parameters in classical dielectric to evaluated the interference effect and study the cut off $\mathrm{k}_{\max }$ in quantum dielectric we arrive:

1- At low velocity the collective interactions are controlled than individual.

2- Coulomb effect appear in low velocity (classical dielectric).

3- Interference effect is very important when the distance between tow ions similar or equal to Debye length $\left(\lambda_{D}\right)$.

4- Contribution of collective modes decreases with temperature and increases with density on shown through the increasing of folds.

\section{Refrences}

[1] Deutsch C., H.B. Nersisyan "Correlated Fast Ion Stopping In Magnetized Classical Plasma", physics.plasma-ph, 1,(1998) 9804029,.

[2] Koskinen M., Lipas P.O., "Electron-Gas Clusters: Tthe Ultimate Jellium Model” Z.physcs. D.35, (1995) 285-297.

[3] Javier F. and Isabel A. "Collective Effects In The Energy Loss Of Large Hydrogen Clusters", Phys.Rev.A 5, 54, (1996).

[4] Nestor R. Aristat and Albert G., "Cluster Stopping Power For an Electron Gas at Finite Temperatures: Calculations For Hydrogen and Water Clusters”, J. Phys.:Condens, 7931-7943 (1991). 
[5] Kadomtsev B.B. “Tokmak Plasma A complex Physical System” Institute of Physics Pablishing Bristol and Philadelphian, (1992).

[6] O'Avanzo J., Lontano M., Bortignon P.F., "Fast-Ion Interaction In Dense Plasmas With TwoIon Correlation Effects", Physical Rev. E 5, 47 (1993).

[7] Pablo T. L, Shalom E., "Fusion Energy In Degenerate Plasmas",Elsvier Physics Letters A 343, 181-189 (2005).

[8] Michael F. “ Scattering Theory” thises, (2008).

[9] Zhang Y., Yuan-Hong S. "Study Of Stopping Power For a Proton Moving In a Plasma With Arbitrary Degeneracy”PHYSICS OF PLASMAS 20,(2013), 102121.

[10] Bringa E.M., Arista N.R. "Energy loss of Correlated Ions In Plasmas: Collective And Individual Contribution" Physical Rev. E, (1996).

[11] Isidro V., Arista N.R. and Rafael G. “Stopping Power And Polarization Induced In a Plasma By a Fast Charged Particle In Circular Motion”. Phys. 35, (2002).

[12] Thomas P. and Jurgen M., "Energy loss of Heavy Ions in Dense Plasma Linear And Nonlinear Vlasov Theory For The Stopping Power", Physical Revl A, 4, 43, (1991).

[13] Deutsch C. and Fromy P., “Correlated Ion Stopping In a Dense Classical Plasma”, Phys.Rev.E, 1, 51,(1995).

[14] Bonitz M.,Pehlke E. and Schoof T.,"Comment On 'Discussion on Novel Attractive Force Between Ions in Quantum Plasmas-Failure Of Simulations Based on a Density Functional Approach”, Phys. Scr.88 (2013).

[15] Schmidt J. A., Thomassen K. I. and Goldston b R. J., "The Design of the Tokamak Physics Experiment (TPX)"Journal of Fusion Energy, 12, 3,(1993).

[16] Shukla P. K. and Akbari-Moghanjoughi M., "Hydrodynamic theory for ion structure and stopping power in quantum plasmas" Physics Rev.E 87, (2013) 043106.

[17] Jacob E. "Fuel Ion Densities and Distributions In Fusion Plasmas" thises (2012).

[18] Smirnov V.P, “Tokmak Foundation In USSSR/Russia 1950-1990”Nucl.Fusion 50,,(2010), 8.

[19] George C. Marshall, "Conceptual Design of a Z-Pinch Fusion Propulsion System" Physics of Plasmas 14, 2. (2007).

[20] Deutsch C. "Ion Cluster Stopping In A Degenerate Electron Plasma" Particle Accelerators, 37,38 (1990) 355-359. 